\title{
LEVELS OF COMPREHENSION OF SCIENTIFIC PROSE: THE ROLE OF TEXT VARIABLES
}

\author{
EDUARDO VIDAL-ABARCA** and VICENTE SANJOSE $\dagger$ \\ *Department of Educational Psychology, University of Valencia, Valencia, \\ Spain \\ $\dagger$ Department of Science Teaching, University of Valencia, Valencia, Spain
}

\begin{abstract}
This research investigates the role played on shallow and deep levels of comprehension by textual changes that are aimed at: (a) improving the relationships within text ideas, and (b) producing better links between text ideas and the reader's knowledge. Four versions of a long physics passage were elaborated combining both kinds of textual changes. Four groups of tenth graders were each given one of the four versions. Different measures representative of these levels of comprehension were taken: getting main ideas, recall, and problem solving. The results indicated that: (a) main idea performance was affected by improving the relationships within text ideas, (b) both textual changes contributed separately to recall, and (c) problem solving increased only when the two changes were presented together. (C) 1998 Elsevier Science Ltd. All rights reserved
\end{abstract}

Scientific texts are an important tool for learning and their improvement is a relevant educational issue. Since the 1980's, many studies have been developed to test the efficacy of certain textual changes. These changes may be classified into two groups: (a) changes aimed at improving the relationships within text ideas without adding new content facts to the original text, and (b) changes aimed at producing better links between text ideas and the reader's previous knowledge by adding new content facts to the text. We may call the former coherence textual changes, and the latter linking textual changes.

Coherence textual changes refer to changes which, firstly, improve global coherence by explicitly including topical expressions such as headings, topic sentences, or summaries, (van Dijk, \& Kintsch, 1983; Kieras, 1985), and secondly, increase connections from one idea to the next in the text. In the former, topical expressions facilitate the processes of macrostructure formation (Meyer, Brandt, \& Bluth, 1980; Britton, Glynn, Meyer, \& Penland, 1982). These processes are especially demanding in scientific texts given that readers generally have little previous knowledge of their content (Britton, Glynn, \& Smith, 1985). In the latter, the

*Departamento de Psicologia Evolutiva y de la Educacion [Department of Developmental and Educational Psychology], University of Valencia, P.O. Box 22045, 46071 Valencia, Spain. E-mail: Eduardo.Vidal-abarca@uv.es 
increase in connections within an existing text can be accomplished in two ways: by re-stating textual ideas, and by making explicit certain implicit relationships presented in the original text.

In general, a re-statement of textual ideas refreshes the memory and aids the reader in her textual understanding (Kintsch, \& van Dijk, 1978; Britton, Van Dusen, Glynn, \& Hemphill, 1990). In particular, scientific texts frequently present a series of premises and then a long chain of reasoning from which the reader must ultimately understand a given conclusion. However the novice reader may have difficulty following that chain of reasoning, especially if the premises were cited so long ago that the information needed to process the conclusion is no longer in his working memory. In these cases, re-statement of premises helps the reader to understand the conclusion.

Making explicit certain implicit relationships by adding conditional or functional expressions, highlights those relationships and lowers text processing demands on the reader. These expressions do not add new content facts to the original text but are crucial in scientific texts which are characterized by their unfamiliarity to the reader.

Linking textual changes implies inserting new content facts to improve the linkage between text ideas and the reader's previous knowledge, (Beck, McKeown, Sinatra, \& Loxterman, 1991; Britton et al., 1990). These changes can be the insertion of additional premises, or the addition of familiar images to link abstract ideas with concrete images.

Additional premises are often required to fill in the gaps frequently found in chains of reasoning which the author may have left out assuming that the reader controls knowledge that she is capable of activating at the appropriate moment. Inserting this information aids comprehension (Beck et al., 1991; Britton et al., 1990).

The addition of concrete images may clarify the abstract information found in scientific texts. Traditionally, analogies are one type of imagery employed with success, (Vosniadou, \& Schommer, 1988; Dupin, \& Joshua, 1989; Halpern, Hansen, \& Riefer, 1990; Duit, 1991).

With linking textual changes, the writer should make explicit only that information that one may reasonably assume will not be easily activated in the comprehension process to avoid that the text became tedious and boring.

Studies aimed at improving scientific text design have considered: (a) coherence textual changes, (e.g., Kintsch, \& Yarbrough, 1982; McNamara, Kintsch, Songer, \& Kintsch, 1996); linking textual changes, (e.g., Vosniadou, \& Schommer, 1988; Dupin, \& Joshua, 1989); and, (c) the two kinds of textual changes simultaneously, (e.g., Beck et al., 1991; Britton, \& Gülgöz, 1991). However an exhaustive literature search does not reveal any study on the combination of the two kinds of textual changes separately and in tandem to test the effect of each possible element and of their combination on different levels of comprehension.

The text comprehension theory of van Dijk and Kintsch (1983), distinguishes between different levels of comprehension to avoid the lack of precision inherent in the term comprehension, (Kintsch, 1994). Apart from the most superficial level, (e.g., encoding words, phrases and the linguistic relations between them), two more levels are distinguished. The semantic and rhetorical structure of the text provides a set of relationships that are important in comprehension and that are frequently encoded. Van Dijk and Kintsch have called this level the textbase and have distinguished it from the situation model which corresponds to a deeper level of understanding. In the situation model, the information provided by the text is elaborated from prior knowledge and is integrated into it (Kintsch, 1994).

The distinction between the textbase and the situation model is particularly relevant in 
scientific text processing. A reader may be able to achieve a shallow level of comprehension, (e.g., getting main ideas or recalling), without getting a deep one, that is, being able to use productively the information in novel environments, (e.g., problem solving).

According to van Dijk and Kintsch (1983), coherence textual changes will mainly affect the shallow level of comprehension, (i.e., textbase), but not necessarily the deep one, (i.e., situation model). Kieras (1985) supports this claim with research results and concludes that " ... it appears that subjects [college students] display a marked ability to comprehend the propositional structure of a passage at a shallow level, and then use this information to identify the important content" (p. 102).

To do so students need help in the form of well written texts that allow them to substitute general comprehension strategies for the knowledge they lack, (Kintsch, 1994). Understanding the propositional structure of a passage also increases recall of textual ideas as hierarchical macrostructures form efficient retrieval systems. Nevertheless, this level of comprehension does not guarantee deep comprehension which only results when text information is integrated with previously assimilated knowledge.

According to van Dijk and Kintsch's definition of the situation model, linking changes should improve deep understanding. However this is not a simple process. They state that "... [textbase] is a necessary station on the way toward the 'situation model"', (van Dijk, \& Kintsch, 1983, p. 343). Thus, linking textual changes might only be effective if implemented together with coherence textual changes.

This claim may be understood if the comprehension result is conceived as a network of propositions, (Kintsch, 1988, 1992). This network is formed by links and nodes coming from the text and from the reader's background knowledge activated by text ideas. If readers form poor textbases, linking textual changes will activate the reader's knowledge but the knowledge will have small and disorganized connections with textual ideas resulting in their poor integration in the reader's background knowledge. However, when readers form good textbases, their knowledge will be broadly connected with a rich and organized set of textual ideas and it is likely that integration will occur.

According to this explanation, adding linking textual changes alone to scientific texts will not substantially improve the recognition of the main ideas in a text as these changes contribute slightly to form the propositional structure of a passage. Though it is true that these changes will improve the reader's recall and contribute to the overall network with the links and nodes supplied by his background knowledge, the combination of both linking and coherence textual changes would construct a better and more integrated network with greater apparent effects. Finally, problem-solving tasks, which require deep understanding, will not be improved with linking textual changes alone. Only the tandem use of both kinds of changes will improve this level of comprehension.

In summary, we formulated three hypotheses:

Firstly, students who are presented a text with coherence textual changes and whose task is to discover the main ideas, will out-perform those who read a text without these changes. The insertion of linking textual changes will not produce significant benefits over the original text.

Secondly, the separate addition of linking and coherence textual changes will improve the student's recall in comparison to the original passage. Applying both changes in tandem will increase the student's performance even further.

And thirdly, the independent insertion of linking or coherence changes will not improve 
the student's problem solving performance. Only inserting both changes simultaneously will permit good problem solving performance. Thus, we hypothesized an interaction effect that would affect problem solving.

Method

Participants

Sixty-seven 10th-grade students from three different high Schools in Valencia, Spain, participated in the study. None had studied the topic of the experimental text, Atomic Models, though all had previously studied the required introductory lesson on Static Electricity necessary to understand the experimental text. The students were randomly separated into four approximately equal groups. Each group received a different version of a text about Atomic Models.

\section{Materials}

The original two-part text was quite similar to a lesson taken from a standard high school physics textbook, and was 1328 words long. The first part described the history of the Dalton, Thomson and Rutherford atomic models. The second part explained the structure of the atomic nucleus (i.e., protons and neutrons), and the concepts of atomic mass and atomic number. The section headings used throughout the passage and the two sections explaining Rutherford's atomic model can be seen in Appendix A. Three revised versions were elaborated: linking, coherence, and coherence-plus-linking.

The linking version. This version contained new content facts added to the original text to produce better links between text ideas and the reader's previous knowledge. We included two main types of ideas: (a) information to facilitate the understanding of important textual conclusions, and (b) familiar imagery. The revisions made on the Rutherford atomic model sections are presented in Appendix A.

We decided to facilitate the comprehension of textual conclusions as they are especially difficult to understand due to the great amount of knowledge (i.e., premises) that must be activated for their real understanding. In the first part of the text, we selected experimental facts related to the discordance in atomic models to identify the important textual conclusions. In the second part of the text important conclusions were identified by analyzing the arguments that different scientists presented in defense of the existence of atomic particles. Textual conclusions were all macropropositions according to the Kintsch and van Dijk (1978) criteria for macrostructure formation.

We then analyzed the premises needed to understand the conclusions. When these premises were absent, we evaluated the student's ability to formulate the missing premises on her own. If it was felt that she was incapacle of doing so, these missing premises were added to the original text.

As shown in Appendix A, we added certain ideas to aid in the understanding of Rutherford's conclusion that the greater part of an atom is empty space. We supplied the idea 
that the atoms of gold are linked one to the other. If atoms of gold had been separated as in a gas, particles could have passed through the space between the atoms but not through the space within the atoms.

We also inserted the premises that, (a) the deviations or regressions of the positively charged particles could be due to the forces of repulsion, and (b) that repulsion was provoked by the presence of a similar positive charge in the atoms of gold. These additions were necessary to understand the existence of a positive charge concentrated in a very small area within the atom, one of the most important conclusions gained from Rutherford's experiment.

We did not elaborate on certain ideas because we considered them easily deducible on the part of the student (e.g., particles of the same sign are repelled outwards, or repulsion is proportional to the distance between charges).

Familiar images were used to introduce analogies between the concrete world and abstract ideas (e.g., the solar system and Rutherford's atomic model; a raisin muffin or scone and Thomson's model; and, a driving license number and the atomic number). Imagery was also raised by the introduction of vivid comparisons based upon the similarity of certain attributes (e.g., the nucleus with respect to the atom as a whole and the size of a dried green pea in the middle of a soccer stadium). Examples of these images can be seen in the Appendix A.

Apart from the two types of ideas just mentioned (i.e., information to facilitate the understanding of textual conclusions, and familiar imagery), a category of miscellaneous ideas was included. To deal with a common misconception about the empty space within an atom, we emphasized that the student should, Take note that when we say "the great empty space", we mean exactly that! Like an immense vacuum in which the electrons are whirling about.

Brief examples of general statements were also included. For example, we reinforced the general claim of Dalton's atomic model that atoms of every chemical element have different mass, with the example that the atomic mass of iron is different from that of sulfur.

In other cases a question-answer format was employed instead of a simple exposition: We can ask ourselves from our knowledge about Static Electricity why an aggregate of protons can be stable in such a small zone when they are all positive and consequently should repel one another. The original statement simply claimed that an aggregation of positive particles, such as protons, should not be stable. All of the changes were made in accordance with previous research in science teaching (Driver, 1988; Roth, \& Anderson, 1989).

The new 1633-word text contained 304 additional words, $36 \%$ of which were to facilitate the understanding of the conclusions drawn by the text, $22 \%$ to the category of miscellaneous ideas, and $42 \%$ as imagery.

The coherence version. This revised version was nearly identical to the original text except for some changes aimed at improving global coherence and increasing connections within text ideas. The revision made on the Rutherford atomic model and its corresponding headings are presented in Appendix A.

Global coherence was improved by means of either changing or including topical expressions in the form of headings and summaries. Headings were to reflect the main topics of the section. Others were added as a preface to the general idea of a particular section. For example, the original heading, The Nuclear Model of the Atom, has nothing to do with the content of the experimental section of Rutherford's model, therefore the title was changed to The Rutherford Experiment and Rutherford's (1871-1937) Conclusions. The title better 
fits the main topics of the section. The connections between the headings and the text macropropositions can be seen in Fig. 1.

Summaries were also inserted. We partially summarized Rutherford's model as a preface to the section on nucleus composition, (see Appendix A). The information on protons and the atomic number was summarized as was that which appeared at the end of the text pertaining to the structure of the atom and the features of atomic particles.

Apart from the above additions, and following Kieras' recommendations (1985), topical or macrostructural expressions were highlighted by means of specific surface structures (e.g., Rutherford's conclusions isolated and printed in bold-face type; see Appendix A). One may also highlight information by initiating a paragraph or section with pertinent information as we did when we placed the paragraph on atomic models as representations of the atom, at the beginning of the first text section instead of at the end of the section on Thomson's atomic model.

Connections within text ideas were improved in two ways: by re-stating ideas to improve the connections between the conclusions and the premises; and, by either changing the order of the ideas or by making explicit certain implicit relationships in the original text. Two clear examples of the former are presented in Appendix A and schematically shown in Figure 1. The information on positively-charged projectiles in Rutherford's experiment was repeated. This information must then be linked to the different trajectories of projectiles, and the conclusion about the concentration of positive charge in a tiny nucleus. A more important example refers to the links that connect Rutherford's conclusions, Rutherford's model and Thomson's model (see Figure 1). In this case, some ideas from Thomson's model were re-stated to make the contradiction between Rutherford's conclusions and Thomson's model more apparent. In this way the essence of Rutherford's model was made more evident.

The second way to improve textual connections is to either change the order of the ideas, or to make explicit certain implicit relationships in the original text. An example of the first procedure was to invert the order of ideas in the opening paragraph on the section about Rutherford's experiment, the purpose of the experiment was presented, (i.e., prove the validity of the Thomson model), and then the procedure was described (see Appendix A). In this way, the explanation of Rutherford's desire to prove the validity of the Thomson model acted as a bridge between the preceding discussion on the Thomson model of the atom and the section treating Rutherford's experiment.

As an example of the second procedure, we introduced the expression this implies that to explicitly link the idea that the greater part of an atom is empty space to the idea that the mass of the atom must be concentrated in a very small area. More importantly, we made explicit the idea that Rutherford was aware of the contradiction between his conclusions and Thomson's model and that this caused him to formulate a new model of the atom (see Figure $1)$.

Apart from the above changes, the information about an experiment on gas discharge was shortened from 36 to 23 words, omitting irrelevant details. The 1666-word coherence version had 338 additional words, $68 \%$ of which corresponded to summaries and headings, $24 \%$ to text re-statements, and $8 \%$ to expressions to make relationships between text ideas explicit.

As a result of these changes, we thought that textual coherence would be improved in the coherence version. To test our assumption, we devised two separate indices similar to a combined measure used by Perrig, and Kintsch (1985). 

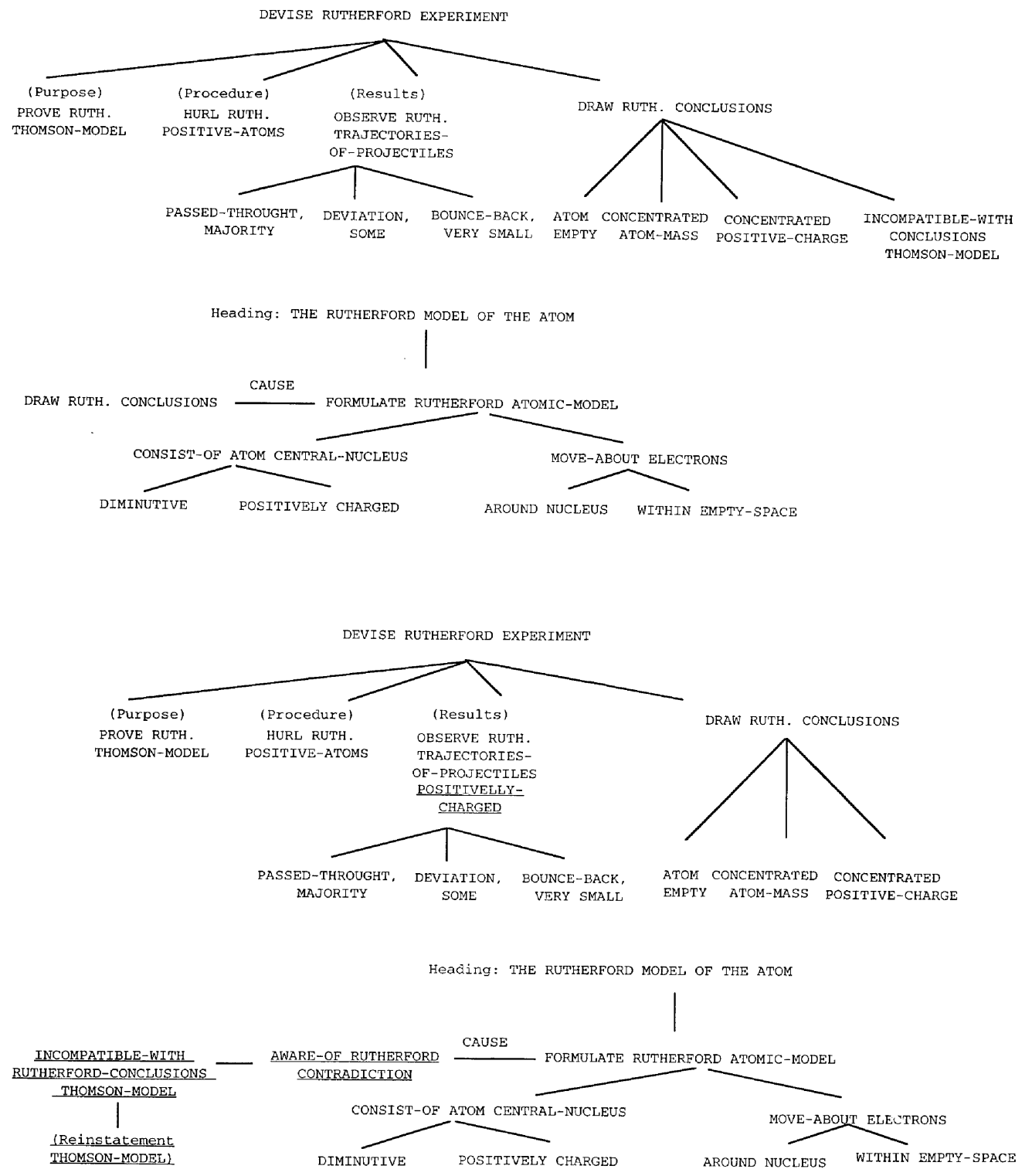

Figure 1. Visual representation of macrostructure for the text sections on Rutherford's atomic model in the original and coherence versions. The propositions re-stated and the explicit connections added in the coherence version are underlined in the representation. 
The text was first divided into idea-units which roughly corresponded to complete sentences (see division in idea-units on the Appendix A). Each idea-unit was then divided into propositions following the procedure of Bovair, and Kieras (1985).

The first index of textual coherence was obtained by counting the number of propositions from previous idea-units that were embedded in any subsequent idea-unit. We suspected that this index would be higher in the coherence version than in the original version given that summaries and text re-statements involved embedding propositions from earlier ideaunits into subsequent ones. We found that the average number of propositions from previous idea-units embedded in subsequent ones was 0.232 for the original version, and 0.645 for the coherence version, $t(147)=2.48, p<.05$.

The second index of textual coherence was obtained in the following manner. The number of times an argument was repeated within an idea-unit was added to the repetitions of the argument in the previous idea-unit. The procedure was repeated with each independent argument presented in a particular idea-unit. Finally, all the figures were added to create a global sum of repetitions for each idea-unit. Then, the index of textual coherence was obtained by summing all the global repetitions, and dividing by the number of idea-units. This is an index of the cohesive elements within an idea-unit, and the relationship of that idea-unit and its preceding idea-unit.

This second index of textual coherence corresponds to the changes aimed at improving connections among textual ideas either by changing the order of the ideas, or by making explicit certain implicit relationships in the original text. The average number of repetitions per idea-unit was 5.014 for the original version, and 6.17 for the coherence passage, $t(147)=2.48, p<.05$. Differences between the coherence and linking versions in the first and second indices of textual coherence above mentioned, were also significant, $t(159)=2.89$, $p<.05$; and $t(159)=2.08, p<.05$, respectively $(M=0.200$ and $M=5.047$ for the first and second indices in the linking version). Differences between the original and linking versions were not significant for either index.

The coherence-plus-linking version. The coherence-plus-linking version included both types of textual changes. Global coherence and connections within text ideas were improved, and information was added to produce a better link between text content and the previous knowledge of the student. The 1984-word version was $49 \%$ longer than the original.

Averages for the first and second indices of textual coherence were 0.557 and 6.090 , respectively. Differences between this version and the two low coherence versions in both indices were significant, though no differences were found between the coherence-pluslinking version and the coherence version.

\section{Procedure}

All tests were administered during the active school year. Students were told that they would be participating in a teaching experiment and that the test results would be taken into account in their school marks. The first day, in a brief session, we tested the student's previous knowledge on the topic. During the second session the students had 20 minutes to read one of the four passages. They were told that after this period of time the text would be removed and that they would be given another 20 minutes to write everything they could 
recall. During the third session students were given the same text version they had read previously and were allowed to use it as a reference while they completed the main idea and problem solving tasks. Each sessions was separated by a period of 2 to 3 days.

\section{Measurement and Scoring}

Previous knowledge test. This is an indirect measure of previous knowledge inspired by other studies in which the student's structural knowledge was assessed (Goldsmith, Johnson, \& Acton, 1991; Schvaneveldt, Durso, \& Dearholt, 1989). In our test, the students had to write between 5 and 10 sentences, using 15 important and repetitive terms or expressions taken from the text: atom, atomic model, atomic number, charge, electron, element, experiment, mass, mass number, matter, nucleus, neutron, particle, proton, Rutherford.

Students were told to use as many different terms as possible. There were no restrictions on the repetition of terms. Sentences could be simple or as complex as students were capable of devising. We then gave examples to the students ilustrating the above points.

The scoring procedure was as follows. First all possible correctly paired-term relationships were calculated. Then one point was given for each correct relationship which the student had included in her sentences. This measure was used as the covariate in our analyses.

The validity of this measure was tested in a pilot study. Significant differences between students who had studied the lesson on Atomic Models and those who had not yet studied it were found.

Immediate recall test. Student recall protocols were scored against the information included in the original passage. We did not consider the information added in the two linking versions because we were interested in testing the effects our changes had over the original passage.

The text was divided into 75 large idea-units. We used idea-units instead of propositions because the text was quite long and we were more interested in the recall of complete thoughts. Each idea-unit corresponded roughly to one complete sentence. Some idea-units were simple sentences, but the greater part were complex (see division in idea-units on the Appendix A).

Twenty-seven of these idea-units were considered as high level idea-units because they contained macropropositions. The rest were low level idea-units. We awarded 1 point if the idea was complete and 0.5 if it was not. Examples of high and low-level idea-units are the following:

(1) High-level idea-unit: Given that the majority of the positively charged particles passed straight through, one must conclude that the greater part of an atom is empty space.

(2) Low-level idea-unit: The quantity of deviations or regressions is controlled by how near or how far the positively charged particles came to the positive charge of the gold atoms.

Main-ideas test. In this task students were given the following instructions:

Here is the text on Atomic Models that you read a few days ago. It contains basic information that a high school student who might not decide to continue on to a higher level course in sciences, might be reasonably 
expected to know. ${ }^{1}$ What is the essential information that the text is trying to get across to this kind of student? Write the 7 or 8 ideas that you would consider the most important when presenting this basic information. Use only the information and ideas presented in the text. Do not bring in any outside information that you might know but may be absent from the text itself.

To clarify the task, the students read a text of approximately 300 words and were then given examples of possible main ideas varying in complexity, elaboratedness, and abstractness.

We asked students to write separate ideas instead of summaries, taking into account Kieras' recommendations and his warning about the pitfalls in writing summaries (Kieras, 1985). We also gave the students a specific goal (7 or 8 sentences) so that they wrote neither too many nor too few sentences. Students' answers were scored against text macropropositions. Each macroproposition present was awarded 1 point.

Problem solving test. In this task, students answered five open questions that measured their skills at applying textual information in new contexts (i.e., problem solving). Each question was awarded points ranging from 0 to 1 . See Appendix B for questions and scoring criteria.

All tests were independently scored by two raters. Interrater agreement ranged form 0.87 to 0.95 .

\section{Results}

The data were analyzed with a 2 (coherence: low, high) $\times 2$ (linking procedures: present, absent) analyses of covariance (ANCOVA) for each dependent measure, taking prior knowledge as the covariate. Apart from main and interaction effects, we were also interested in certain comparisons between pairs of text versions, which were relevant to our hypotheses. These pre-planned comparisons were made by using multiple contrasts between means by the Bonferroni method. Unless otherwise stated, all reported analyses are reliable with a probability less than 0.05 . Means and standard deviations of all the tests can be seen in Table 1.

Main-ideas Test. Previous knowledge was a significant covariate, $F(1,62)=7.858$. Only the main effect of coherence was reliable, $F(1,62)=17.010, M S E=20.023, p<.001$. Multiple contrasts between means showed that the coherence version had significant advantages over the original, but not over the linking version. However, the coherence-plus-linking version was superior to the original and to the linking versions, but not to the coherence version, as we had predicted. Coherence appears to be the most important variable in understanding the main text ideas, though linking procedures produced more benefits than we had first thought.

Immediate Recall Test. We distinguished between recall of high and low level idea-units. In recall of high level idea-units the covariate was not significant. Main effects of coherence and of linking procedures were significant, $F(1,62)=17.989, M S E=25.188$, and

\footnotetext{
In the Spanish school system, all high school students take a basic science course in which the lesson on Atomic Models is included. It is then up to the student to decide whether he will continue on to a higher level course or not.
} 
Table 1

Results on the dependent measures

\begin{tabular}{|c|c|c|c|c|c|}
\hline \multirow[b]{2}{*}{ Group version } & & \multirow[b]{2}{*}{ Main ideas } & \multirow[b]{2}{*}{ Problem solving } & \multicolumn{2}{|c|}{ Recall of idea-units } \\
\hline & & & & High level & Low level \\
\hline \multirow[t]{3}{*}{ Original } & $n=17$ & & & & \\
\hline & $M$ & 8.35 & 1.71 & 5.71 & 3.36 \\
\hline & $S D$ & 3.90 & 1.05 & 3.50 & 2.00 \\
\hline \multirow[t]{3}{*}{ Linking } & $n=18$ & & & & \\
\hline & $M$ & 11.78 & 2.14 & 9.50 & 4.42 \\
\hline & $S D$ & 4.62 & 1.12 & 6.10 & 2.90 \\
\hline \multirow[t]{3}{*}{ Coherence } & $n=15$ & & & & \\
\hline & $M$ & 14.00 & 2.35 & 9.80 & 3.53 \\
\hline & $S D$ & 5.03 & 1.39 & 4.60 & 2.80 \\
\hline \multirow[t]{3}{*}{ Coh-plus-Link } & $n=17$ & & & & \\
\hline & $M$ & 15.12 & 4.00 & 15.71 & 5.71 \\
\hline & $S D$ & 5.23 & 1.07 & 5.40 & 3.60 \\
\hline
\end{tabular}

$F(1,62)=14.267, p<.001$, respectively. The interaction effect was not significant. Each variable seems to produce independent and additive effects in the coherence-plus-linking version.

This effect can be seen when the means of the revised versions are compared with the original. The linking version added nearly 4 points to the original (from 5.71 to 9.50 ); the coherence version produced an advantage of 4 points (from 5.71 to 9.80); and the coherenceplus-linking caused a benefit of 10 points (from 5.71 to 15.71). Pre-planned multiple contrasts between means showed that the coherence-plus-linking version had significant advantages over all other conditions. Neither the coherence version nor linking version were significantly superior to the original. These results coincide with our predictions.

Results of the recall of low level idea-units were quite different from those above mentioned. The covariate reached a significant value, $F(1,62)=7.444, p<.01$. Coherence did not yield a significant main effect, and linking procedures were very close to the usual acceptable level, $F(1,62)=3.902, M S E=7.568, p=.053$. If we compare the means, we see that the addition of linking procedures to the original version contributed slightly to the recall of low-level ideas. Improving text coherence did not produce any advantage either; but, when both changes were used in tandem, the students' performance improved, though differences were not significant. These results clearly contradict our predictions.

To examine in greater detail the effects that each of the textual changes had over recall, we performed two complementary analyses: (a) the percentage of idea-units emphasized by topical expressions; and, (b) the percentage of idea-units accompanied by additional information (see Table 2). Both (a) and (b) represent percentages based upon total idea-units recall. The data were analyzed with a 2 (coherence) $\times 2$ (linking procedures) analysis of variance (ANOVA). With respect to the percentage of idea-units emphasized by topical expressions, only the main effect of coherence was significant, $F(1,62)=7.572, p<.01, M S E=415.808$. Multiple contrasts between means showed that the two coherence versions had significant advantages over the original version. In the percentage of idea-units accompanied by additional information, the only significant effect came from the linking procedures, $F(1,62)=4.051, M S E=190.534$. Bonferroni's test showed that the linking version had significant advantages over coherence. So, it would appear that each textual change has a specific effect on recall. 
Table 2

Percentage of highlighted idea-units over the total idea-units recall

\begin{tabular}{llcc}
\hline \multirow{2}{*}{ Group version } & & \multicolumn{2}{c}{ Highlighted idea-units by } \\
Original & $M$ & Topical expressions & Additional information \\
\hline \multirow{2}{*}{ Linking } & $S D$ & 44.33 & 40.36 \\
& $M$ & 21.70 & 13.52 \\
Coherence & $S D$ & 51.35 & 49.21 \\
& $M$ & 18.25 & 18.33 \\
Coh-plus-Link & $S D$ & 63.10 & 35.95 \\
& $M$ & 25.40 & 10.81 \\
\hline
\end{tabular}

Problem Solving Test. The previous knowledge score was also a significant covariate, $F(1,62)=21.914$. Main effects of coherence and of linking procedures were reliable, $F(1,62)=26.474, M S E=1.251$, and $F(1,62)=14.516, p<.001$, respectively. The interaction effect was also significant, $F(1,62)=4.424$. Thus, the separate insertion of linking or coherence changes had a slight effect on the improvement of the student's problem solving performance, but the combination of both changes in tandem produced a significant advantage. Bonferroni's test showed that the coherence-plus-linking version had significant advantages over all the other passages, but any other difference was significant. These results coincided with our predictions.

\section{Discussion}

Our first hypothesis predicted that students who were presented with a text with coherence textual changes and who had to discover the main ideas, would out-perform those who read a text without these changes. We had also predicted that the insertion of linking textual changes would not produce significant benefits over the original text in this task. The experimental results confirm this hypothesis, and they may be explained by considering the effects of the topical expressions. Coherence versions were organized in such a way that they presented powerful cues to the reader about the macrostructure: headings, initial position, bold print, and lead or initial thematic sentences

The positive effects of these changes on macrostructure formation are well documented (van Dijk, \& Kintsch, 1983; Kieras, 1985). Thus, it seems that main ideas can be derived with only shallow semantic knowledge, and through the use of textual and sentence surface structures as well as the propositional content.

In our second hypothesis, we expected that the separate addition of linking and coherence textual changes would improve the student's recall over the original passage, whereas applying both changes in tandem would increase the student's performance more dramatically. This hypothesis is confirmed in the high-level idea-unit recall measurement, though not in low-level idea-unit recall. It would appear that just coherence or just linking textual changes improve high-level idea-unit recall even though the differences with respect to the original text are not significant. However those differences were definitely significant between the coherence-plus-linking text and the rest of the versions.

According to the Kintsch model $(1988,1992)$, the effect that certain textual changes have 
on the network that a reader forms as a result of comprehension, may be one explanation for the above results. The changes in coherence contribute towards a greater number and better organization of the nodes and links inherent in the text (i.e., the textbase). The linking changes contribute to the network through the activation of ideas from the reader's own background knowledge. When both changes are introduced in tandem, it appears that the positive effects combine additively.

The independent effect of each kind of change is confirmed by the complementary analyses. When we analyze the percentage of idea-units highlighted by topical expressions, (i.e., high-level idea-units) over the total number of idea-units, we find that only the coherence version makes a significant contribution. If we pay close attention to the percentage of recall of idea-units accompanied by additional information, it will be noted that it was the linking variable that produced significant differences. That is, it seems that both kinds of changes contribute to the remembrance of the ideas that they themselves highlight whether via topical expressions or via analogies and re-statements.

On the other hand, results in recall of low level idea-units are quite different. The addition of linking procedures to the original version contributed only slightly to improved scores, and coherence textual changes did not produce any advantage. When both changes were put together, student performance improved, though differences were not significant. These results may be explained by considering that the coherence textual changes highlighted high level ideas and that they may have made students pay less attention to low-level information. On the other hand, some researchers have found low scores in the recall of low-level information when the content of the text is unfamiliar to the reader and the text is long (McNamara et al., 1996).

Results fully confirm our third hypothesis. The independent insertion of linking or coherence changes did not improve the student's problem solving performance, and only by inserting both changes together was good problem solving performance achieved. We have found the interaction effect between both changes that we had predicted. This result is in agreement with the van Dijk and Kintsch model (1983).

Problem solving performance requires deep comprehension, and is the result of a good situation model. Forming such a mental representation demands the integration of text information into the reader's background knowledge. This integration will likely occur only when readers can first form good textbases by building a rich and organized network of textual ideas. Then, the knowledge that readers activate will be broadly connected with the network. Therefore, the likelihood that integration will occur is high. Otherwise, activated knowledge will have small and disorganized connections to the text ideas, and it will result in poor integration. Thus, constructing textbases is a necessary stage on the way towards the situation-model formation.

Results have been reported showing that readers may form good situation models with poor textbases (Bransford, Barclay, \& Franks, 1972; Garnham, 1981). However when the conditions described in these studies are considered, one notices that they are quite different from those given to the reader of scientific texts. Not only were the texts used by the above cited authors limited to paragraphs of three or four sentences and comprised of familiar material, but the reader was also asked to do a different task (i.e., complete a recognition task comparing pairs of sentences with minimal distinction in the textbase but great distinction in the situation model). Under these conditions it is possible to 
construct a good situation model without having first developed a correct textbase. We do not believe this is acceptable nor possible for a reader of scientific texts under normal conditions.

The results of a study by Beck, McKeown, and Worthy (1995), might be taken as affirmation of this point. The purpose of Beck and her colleagues was to examine the effects on student comprehension of what they called voiced versions of a more and less coherent text. Voiced passages connect text information to the reader's background knowledge via linguistic procedures by adopting a conversational tone, addressing the reader directly, portraying the immediacy of events and reactions of human agents (e.g., in history texts), and so on.

We believe that voiced procedures have the same function as our method of linking. What these researchers found was that when these linguistic procedures where added to a poorly coherent text, the deep comprehension of the text was in no way improved. The improvement occurred only when the linguistic methods were inserted into a text with a high level of textual coherence.

To conclude, we would like to comment on the designing of scientific texts. In writing a science text, our goal should be to allow the reader to create proper models of the situation described or explained in the text, not only to provide easy readability and fluent recall (Kintsch, 1994). The point is that we know much more about the latter than the former. Moreover, the guidelines to improve the textual coherence are quite general and can be applied with relative ease (e.g., introducing topical expressions, re-stating information, etc; see also the computational method by Britton, \& Gülgöz, 1991).

However there are no clear procedures for how to improve the integration of text information into the understanding of the reader. These procedures depend greatly on the background of the reader and on the content of the text. Thus, the linguistic methods employed by Beck et al. (1995), might be adequate for fourth graders, but not for high school students. Similarly, it might be adequate for text on history but not for text on other content areas.

Whatever the case, a general procedure to improve the linkage between text information and the reader's previous knowledge might consist in the following steps: (a) to identify the textual macropropositions as textual changes should mainly affect this information; (b) to analyze if possible readers may have the knowledge they might need to deeply understand the textual macropropositions, or if it is necessary to add some content ideas into the text; (c) to examine if the textual information, and especially text macropropositions, can be brought closer to the readers by introducing imagery, or by using linguistic resources (e.g., Beck et al. procedures). This general procedure may contribute to improve deep level understanding. Future research will consist in testing this procedure on different texts from different content areas.

Acknowledgments-Earlier versions of this paper were written by the first author during a stay at the Center for the Study of Reading (University of Illinois at Urbana-Champaign) supported by a grant from Spanish Subdireccion General de Investigacion Cientifica y Tecnica (no PR94-144). We thank Bill Nagy, Tom Anderson, Isabel Beck, Moddy McKeown, and two anonymous reviewers for their helpful comments on earlier versions of the manuscript.

\section{References}

Beck, I. L., McKeown, M. G., \& Worthy, J. (1995). Giving a text voice can improve students' understanding. Reading Research Quarterly, 30, 220-238. 
Beck, I. L., McKeown, M. G., Sinatra, G. M., \& Loxterman, J. A. (1991). Revising social studies text from a textprocessing perspective: evidence of improved comprensibility. Reading Research Quarterly, 26, 251-275.

Bovair, S., \& Kieras, D. E. (1985). A guide to propositional analysis for research on technical prose. In B. K. Britton, \& J. B. Black (Eds.), Understanding expository text (pp. 315-362). Hillsdale, NJ: Lawrence Erlbaum Associates.

Bransford, J. D., Barclay, J. R., \& Franks, J. J. (1972). Sentence memory: A constructive versus interpretive approach. Cognitive Psychology, 3, 193-209.

Britton, B. K., Glynn, S. M., Meyer, B. J. F., \& Penland, M. J. (1982). Effects of text structure on use of cognitive capacity during reading. Journal of Educational Psychology, 74(1), 51-61.

Britton, B. K., Glynn, \& Smith, J. W. (1985). Cognitive demands of processing of expository text: A cognitive workbench model. In B. K. Britton, \& J. B. Black (Eds.), Understanding expository text (pp. 227-248). Hillsdale, NJ: Lawrence Erlbaum Associates.

Britton, B. K., \& Gülgöz, S. (1991). Using Kinstch's computational model to improve instructional text: Effects of repairing inference calls on recall and cognitive structures. Journal of Educational Psychology, 83(3), 329-345.

Britton, B. K., Van Dusen, L., Glynn S. M., \& Hemphill, D. (1990). The impact of inferences on instructional text. In A. C. Graesser \& G. H. Bower (Eds.), The psychology of learning and motivation (25; pp. 53-70. San Diego, CA: Academic Press.

Driver, R. (1988). Un enfoque constructivista para el desarrollo del curr'culo en ciencias [A constructivist view for Science curriculum development]. Ense-anza de las ciencias, 6, 109-120.

Duit, R. (1991). On the role of analogies and metaphors in learning science. Science Education, 75, 649-672.

Dupin, J. J., \& Joshua, S. (1989). Analogies and modeling analogies in teaching: some examples in basic electricity. Science Education, 73, 207-224.

Garnham, A. (1981 (1991). Mental models as representations of text. Memory \& Cognition, 9, 560-565.

Goldsmith, T. E., Johnson, P. J., \& Acton, W. H.. Assessing structural knowledge. Journal of Educational Psychology, $83,88-96$.

Halpern, D. F., Hansen, C., \& Riefer, D. (1990). Analogies as an aid to understanding and memory. Journal of Educational Psychology, 82(2), 298-305.

Kieras, D. E. (1985). Thematic processes in the comprehension of technical prose. In B. K. Britton \& J. B. Black (Eds.), Understanding expository text (pp. 91-107). Hillsdale, NJ: Lawrence Erlbaum Associates.

Kintsch, W. (1988). The role of Knowledge in discourse comprehension: A construction-integration model. Psychological Review, 95(2), 163-182.

Kintsch, W. (1992). A cognitive architecture for comprehension, In H. L. Pick Jr., P. Van Den Broek, \& D. C. Knill (Eds.), Cognition: Conceptual and methodological issues (pp. 143-163). Washington, DC: American Psychological Association.

Kintsch, W. (1994). Text comprehension, memory, and learning. American Psychologist, 49(4), 294-303.

Kintsch, W., \& van Dijk, T. A. (1978). Toward a model of text comprehension and production. Psychological Review, $85,363-394$.

Kintsch, W., \& Yarbrough, J. C. (1982). Role of rhetorical structure in prose comprehension. Journal of Educational Psychology, 74, 828-834.

McNamara, D. S., Kintsch, E., Songer, N. S., \& Kintsch, W. (1996). Are good texts always better? Interactions of text coherence, background knowledge, and levels of understanding in learning from text. Cognition and Instruction, 14(1), 1-43.

Meyer, B. J. F., Brandt, D. M., \& Bluth, G. J. (1980). Use of top level structure in text: Key for reading comprehension of ninth-grade students. Reading Research Quarterly, 1, 72-102.

Perrig, W., \& Kintsch, W. (1985). Propositional and situational representations of text. Journal of Memory and Language, 24, 503-518.

Roth, K., \& Anderson, Ch. (1989). Promoting conceptual change learning from science textbooks. In P. Ramsden (Ed.), Improving learning (pp. 109-141). London: Kogan Page.

Schvaneveldt, R. W., Durso, F. T., \& Dearholt, D. W. (1989). Network structures in proximity data. In G. H. Bower (Ed.), The psychology of learning and motivation (24, pp. 249-284. New York: Academic Press.

van Dijk, T.A., \& Kintsch, W. (1983): Strategies of discourse comprehension. New York: Academic Press.

Vosniadou, S., \& Schommer, M. (1988). Explanatory analogies can help children acquire information from expository text. Journal of Educational Psychology, 80(4), 524-536.

\section{Appendix A}

Excerpts corresponding to the sections on Rutherford's atomic model for the original, linking, coherence and coherence-plus-linking versions. The original and linking passages are superimposed first, and then the same is done with coherence and coherence-plus-linking passage. Text added in the two versions with linking textual changes is underlined. Numbers in the 
parentheses are the numbers of the idea-units corresponding to each sentence. They were omitted in the versions read by the students.

\section{Atomic models (original and linking versions)}

\section{Electricity and the Atom}

THE THOMSON MODEL OF THE ATOM (1856-1940) THE NUCLEAR MODEL OF THE ATOM

Rutherford devised an experiment in which he stripped atoms of their electrons and hurled the tiny positively charged atoms at a leaf of gold foil that was a thousand times finer than the thickness of a razor blade (25). It occurred to him to prove the validity of Thomson's model by means of this experiment (24). See Fig. 2 below.

Once the atoms collided with the foil, the experiment revealed three basic trajectories of the projectiles (26): the majority passed right through the foil (27); some experienced a slight deviation (28); and, a very small number bounced right back (29). Rutherford found this last trajectory surprising (30). In his own words: "It's like shooting a pistol at a piece of paper and having the bullet come right back and doing you damage." See Fig. 2.

Based upon the results of the experiment Rutherford drew the following conclusions (31) which were incompatible with Thomson's Model (36).

Given that the majority of the particles passed straight through and that the gold atoms are linked one to the other, one must conclude that the greater part of an atom is empty space (32). The mass of the atom must be concentrated in very small area (33). The deviations or regressions of the positively charged particles could be due to the forces of repulsion provoked by the presence of a similar positive charge in the gold atoms. The quantity of deviations or regressions is controlled by how near or how far the positively charged particles came to positive charge of the gold atoms (34). Given the greater number of deviations to regressions, the positive charge in the atom must also be concentrated in a very small area (35).

\section{The Rutherford model of the atom}

Based upon his conclusions, Rutherford formulated a new model of the atom (37). According to the model, the atom consisted of a diminutive central nucleus of great mass and positively charged (38) surrounded by electrons moving about within the great empty space around the nucleus (39). Take note that when we say "the great empty space", we mean exactly that! Like an immense vacuum in which the electrons are whirling about. We are dealing with what is known as a dynamic model (40) in which the movement of the electrons might be likened to the movement of the planets in their orbits around the sun. With that idea in mind the model was called the "Planetary Model". See Fig. 3.

Rutherford himself measured the radius of the nucleus from the data of the experiment (41). He found it to be approximately $10^{-15} \mathrm{~m}(42)$. The radius of the atom as a whole was already known to be $10^{-10} \mathrm{~m}(43)$. Thus the nucleus is some 100000 times smaller than 
the atom as a whole (44). This might be a bit easier to visualize if we were to take the atom as the size of a soccer stadium, then the nucleus would be like a dried green pea down there in the middle of the field.

2. The Nucleus of the Atom

\title{
CLOSER TO THE NUCLEUS
}

Atomic models (coherence and coherence-plus-linking versions)

\section{Atomic Models as Representations of the Atom}

\author{
THE DALTON MODEL OF THE ATOM AND THE PROBLEM OF CHARGE IN \\ THE ATOM \\ THE THOMSON MODEL OF THE ATOM (1856-1940) \\ THE RUTHERFORD EXPERIMENT (1891-1937), AND HIS CONCLUSIONS OVER \\ THE RESULTS.
}

It occurred to Rutherford to prove the validity of Thomson's model (24). He devised an experiment in which he stripped atoms of their electrons and hurled the tiny positively charged atoms at a leaf of gold foil that was a thousand times finer than the thickness of a razor blade (25). See Fig. 2 below.

Once the atoms collided with the foil, he observed three basic trajectories of the positively charged projectiles(26): the majority passed right through the foil (27); some experienced a slight deviation (28); and, a very small number bounced right back (29). Rutherford found this last trajectory surprising (30). In his own words: "It's like shooting a pistol at a piece of paper and having the bullet come right back and doing you damage."

Based upon the results of the experiment Rutherford drew the following conclusions (31):

a) Given that the majority of the positively charged particles passed straight through and that the gold atoms are linked one to the other, one must conclude that the greater part of an atom is empty space (32). This implies that the mass of the atom must be concentrated in a very small area (33).

b) The deviations or regressions of the positively charged particles could be due to the forces of repulsion provoked by the presence of a similar positive charge in the gold atoms. The quantity of deviations or regressions is controlled by how near or how far the positively charged

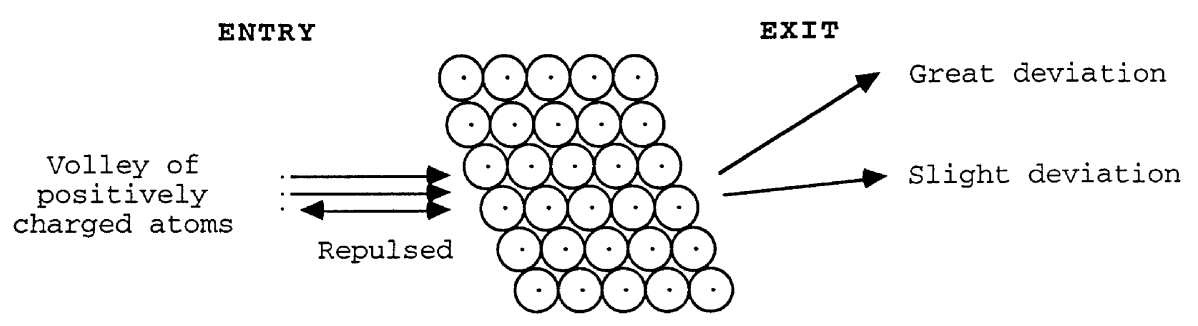

Figure 2. Graphic representation of Rutherford's experiment. 
particles came to positive charge of the gold atoms (34). Given the greater number of deviations to regressions, the positive charge in the atom must also be concentrated in a very small area (35).

\section{The Rutherford model of the atom}

As you can see, these conclusions are incompatible with Thomson's atomic model (36) that mass and positive charge were distributed throughout the atomic volume in which there were neither holes, nor positive charges were concentrated. Rutherford was aware of the contradiction and formulated a new model of the atom (37). According to the model, the atom consisted of a diminutive central nucleus of great mass and positively charged (38) surrounded by electrons moving about within the great empty space around the nucleus (39). Take note that when we say "the great empty space", we mean exactly that! Like an immense vacuum in which the electrons are whirling about. We are dealing with what is known as a dynamic model (40) in which the movement of the electrons might be likened to the movement of the planets in their orbits around the sun. With that idea in mind the model was called the "Planetary Model". See Fig. 3 below.

Rutherford himself measured the radius of the nucleus from the data of the experiment (41). He found it to be approximately $10^{-15} \mathrm{~m}$ (42). The radius of the atom as a whole was already known to be $10^{-10} \mathrm{~m}$ (43). Thus the nucleus is some 100 thousand times smaller than the atom as a whole (44). This might be a bit easier to visualize if we were to take the atom as the size of a soccer stadium, then the nucleus would be like a dried green pea down there in the middle of the field.

2. The Composition of the Nucleus of the Atom.

THE SUBATOMIC PARTICLES

We have just seen how Rutherford introduced a new nuclear model of the atom, i.e., a positively charged nucleus and electrons with the same magnitude of negative charge.

THE PROTON

MENDELEEV AND THE PERIODIC TABLE OF ELEMENTS, AND MOSELEY'S EXPERIMENT

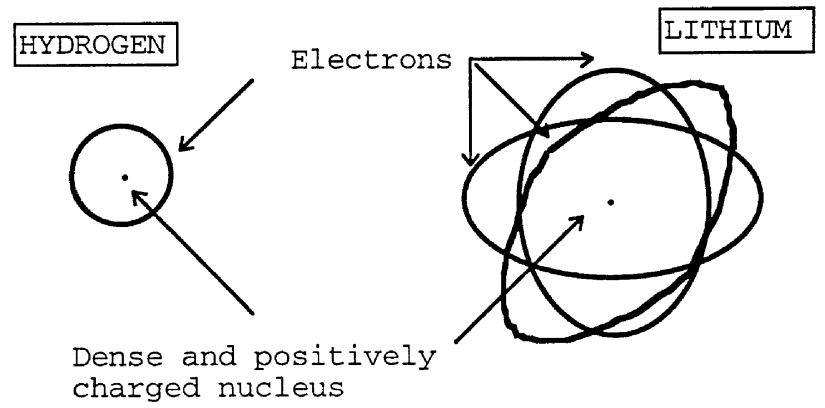

Figure 3. The Rutherford Model of the Atom for a hydrogen and a lithium atom. 


\title{
A NEW ORDER FOR THE ELEMENTS BASED UPON THE NUMBER OF PROTONS: THE ATOMIC NUMBER \\ THE NEED FOR A "NEW" SUB-ATOMIC PARTICLE: THE NEUTRON ATOMIC REPRESENTATION: ATOMIC MASS AND ATOMIC NUMBER
}

\author{
Appendix B \\ Questions and scoring criteria for the problem solving test
}

1. Write down the subatomic particles present in this atom:

${ }_{13}^{27} \mathrm{Al}$

Scoring criteria: 0.33 points for responding correctly to each part of the subatomic particles.

2. Why are protons more difficult than electrons to add to, or remove from, an atom?.

Scoring criteria: 1 for the answer: because the electrons are on the outside; 0.5 points for one of the following answers: because the electrons are lighter, because the nucleus is more difficult to break.

3. If Rutherford had directed negative particles to the sheet of gold instead of positive ones, and the results had been the same, what atomic model would you propose?.

Scoring criteria: 0.5 points for each one of the following answers: the nucleus will be negative; and, the positive particles will be on the outside.

4. Making use of the atomic model you have just proposed, how would you explain electrification by rubbing?

Scoring criteria: it would be the positive charges that would be lost.

5. Would you say that an atom with 6 protons, 6 electrons and 6 neutrons, and another one with 6 protons, 5 electrons and 8 neutrons are atoms from the same chemical element? Why?

Scoring criteria: 1 point for the answer: Yes, because they have the same number of protons (or the same atomic number); 0.5 points for one of the following answers: No, because they don't have the same atomic number, though they have equal number of protons or, No, because they don't have the same mass number even thought hey have the same atomic number. 